\title{
Research on Modern Sports Training Method Based on Computer Virtual Technology
}

\author{
Li Zhishuang \\ School Attached to Shijiazhuang Tiedao University, Shijiazhuang, Hebei, China, \\ 050000 \\ lizhishuang123@163.com
}

\begin{abstract}
Under the background of the fast development of computer industry and with the virtual technology being widely used, information technology in China has made significant progress; however, sports information teaching is still in a relatively tow tevel. Now the application of information technology in physical training has become a big issue which needs to be improved. On the basis of forward kinematics theory and inverse kinematics theory of human engineering and by collecting three-dinensional human body motion information, three-dimensional human body motion model which include the spine movement, arm movement, step movement and the whole human body movement has been built up and simulation experiment on the human body has been conducted. After virtual technology being applied to phyical training field, a conclusion that body movement will be much improved due to the application of virtual technology can be drawn. The effect from high to low is obvious: the most positive effect is on step movement, then arm movement, the whole body movement as well as spine movement. In general, the application of virtual technology in modern physical training field promotes the development of sports career and also expands the applications of computer technology.
\end{abstract}

Keywords: virtual technology, physical training, human engineering, three-dimensional human body motion nodel, human body simulation

\section{Introduction}

Virtual technology, as a synonym for the application of high technology of the combination of human and machine, is a comprehensive method of a interdisciplinary which can transform be physical objects into theoretical construct. It breaks the obstacle of time and space and makes customers have an immersive experience which has unparalleled meaning. The related technology which is familiar to people includes Route, firewall and VPN etc. [1] Computer virtual technology developed under the background of the increasing development of computer technology and virtual technology. [2] On the basis of quantitative analysis of sport technique, computer virtual technology showed the resul by using graphical approach, namely, transforming the basic movement into video by computer technology and then adding comments on it, the teaching work is finished. By virtue of its specific serviceability which includes information management ability, data analysis ability, image editing ability, audio and video collecting ability as well as the sensor application ability, and combined with their advantages which include perceptibility, interactivity, imagination and immersive ability, computer virtual technology played a very significant role in many aspects and fields. [3] With the increasingly rapid pace of modern life, physical quality has attracted more and more attention; however, people don't have an overall understanding on physical training. Besides, due to the lack of normalization in coaching skill and physical facilities and fields, the development and application of computer virtual technology is much needed. 
In this paper, the method of modern sports training based on computer virtual technology is studied which aiming at achieving the optimal effects of physical exercise within the shortest time. Creating a new ways of learning virtual technology and achieving the goal of bringing impetus for Chinese sports training, a new training system needs to be built up thus making a contribution to our country's sports education.

\section{The Application of Modern Sports Training Based on Computer Virtual Technology}

On the application of virtual technology in the current sports teaching, virtual technology conforms to the current development trend of modern sports teaching and meets the requirements of students and users of teaching methods as well as achieving the goal of the interconnection between the coaches and the users. [4] Meanwhile, the application of virtual technology in sports can meet the requirement of the students of different majors besides the knowledge and applying ability of social people of different kinds of work has been largely improved.

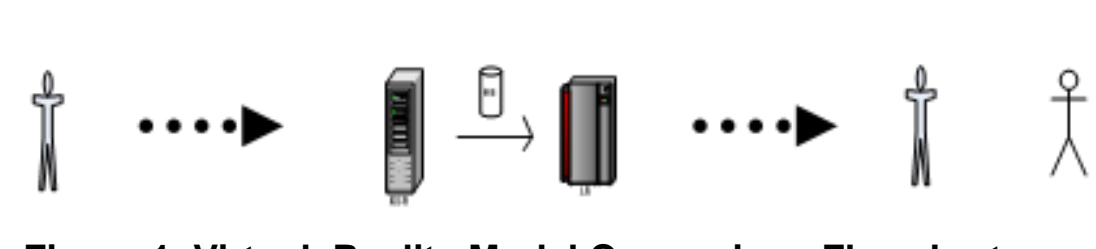

Figure 1. Virtual, Reality Model Comparison Flowchart

From the above figure we can know that the datagenerated by the movement of the human body is input into the computer memory by means of related software. The information stored in the database is transformed to a realistic human motion model, which is displayed on the displa [5] By comparing the real human body movement model with the virtual standard human body model different problems can be found out in the process of physical training, and then such problems can be solved in time. In the comparison of real human motion with yirtual human motion, there are three kinds of environment: reality vs. virtual envitonment, virtual vs. virtual environment and surreal environment. It can be shown as folloys.

\subsection{The Inportance of Computer Virtual Technology to the Application of Modern Sports Training}

Modern sports traing is faced with the problem of low training quality, time deficiency, poor enthusiasm as well as unsound guarantee evaluation system. Under this circumstance, avoiding emergency and preventing physical damage on muscles and bones and nerves due to the difficult movements and changing the unchangeable factors in sports training have become the primary task of the training for instructors. [6] In order to optinize the effect of modern sports training and improve the enthusiasm of social workers, teachers and students to participate in sports activities, in consideration of the importance of ergonomics in health and safety issues, the knowledge and skills of human simulation is more and more extensively applied to human life and product life cycle. [7] By virtue of the information acquisition ability of computer virtual technology and by using computer own capacity for technical analysis, by simulating different kinds of gestures, language, vision and action, it provides a more objective and more accurate theoretical guidance and practical experience for modern sports training. 


\subsection{The Means of Application of Computer Virtual Technology in Modern Sports Training}

(1) Virtual and reality contrast

The Contract between virtual and reality is conducted by computer virtual technology and on the basis of human simulation of physical training activities, and the study of human body movement even to the specific analysis of each gesture which aiming at finding the defect spots among each movement. In later practice, sportsmen have comprehensive understanding for specified actions and they can constantly correct and improve these disadvantages. By giving full play to the advantages of information, the thinking ability of sportsmen can be stimulated thus fully taking the advantage of the functional role of each sensory system

(2) Virtual and virtual contrast

Virtual and virtual contrast, on the one hand uses the virtual technology of computer software to find the training model, on the other hand, inputs the actual movement into computer thus forming action models. After that, by comparing the two, the gap can be found. For the disparity between the two, a clear training scheme is need to be made which aims at showing the direction of the user's training thus to improve the efficiency of the body's training..

(3) Constructing the surreal environment

Surreal environment does not exist in reality instead it is a virtual, varied, dynamic, multiple simulation environments. A number of different environments in different time periods have been created in order to study therinfluence of different environment on the modern sports training. By taking advantages its own unique interactivity of computer virtual technology, such means can enable atbletes to have a real experience to the virtual world, thus realizing remote interactive training. It intend to reduce the effect of the main venue on sports training and reduce the environmental impact on the training.

\subsection{The Application Status of Compuiter Sirtual Technology in Modern Sports Training}

(1) Status at abroad

United States has earlier illustrated the associative relationship between information technology and teaching and integrated measures on information and teaching has been widely conducted [8] On the basis of guaranteeing the teaching, they have vigorously increased investment in in ormation technology infrastructure which aims to strive to complete the various knowledge training program; British put computer information technology into various fields, especially by increasing more funds to improve teachers' quality thus to achieve the high quality of education and training; in Australia, information applications mainly reflected in the users' self-using of information teaching mode, rather than a fixed set. It highlights the personalized arrangements which are not affected by the integral control and impact.

(2) Domestic status quo

Virtual physical analysis system is formed by the combination of computer virtual technology and modern physical training. China has made a series of research theories which include the construction of the teaching content system of computer science in sport in physical education, the construction of experiment teaching system of sports information technology which based on the training mode.

By combining the graphics, images, text and data in sports training, the application fields of the technology and the computer virtual technology in sports training are as follows: 


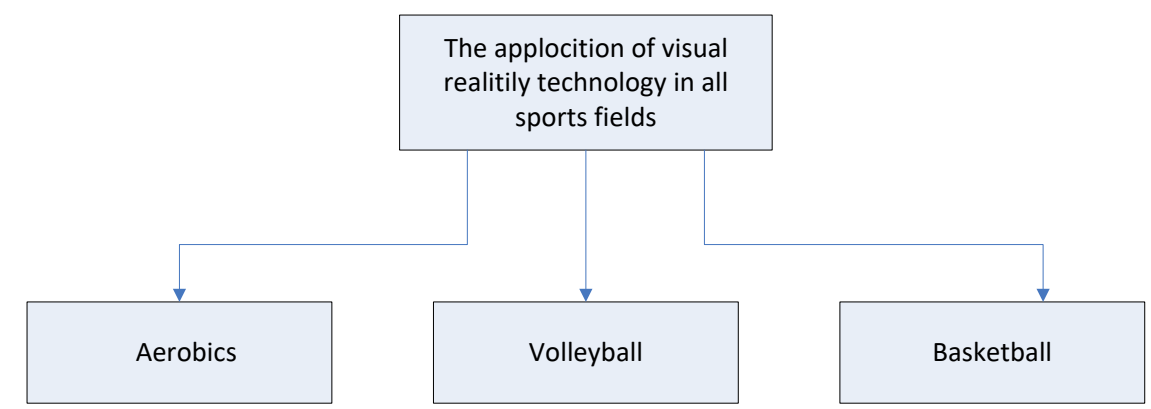

\section{Figure 2. The Application of Visual Reality Technology in All Sports Fields}

In calisthenics teaching training, computer virtual technology is used to stimulate the teaching action and with the coach demonstrating the correct action, calisthenics teaching can be guaranteed. Simulation training provides a more intuitive guidance which is helpful in correcting the wrong action and giving the correct guidance.

In volleyball teaching and training application, computer virtual technology is used to simulate overhand serve, slap, jumping and volleyball movement transmission etc. By stimulating different environments in different areas, time and in different games, physical coordination ability has been improved. The technical novement explanation can be done while observing the movement of volleyball.

In basketball teaching and training application by using computer simulation technology application and based on the best, fastest and moderate speed of athletes, the whole activity process can be controlled. So different training schemes can be designed for different athletes thus ensures that athletes can adjust their own conditions, under this circumstance, athlete's physical qualities ean be improved.

\subsection{The Advantages Disadvantages and Opportunities of Computer Virtual Technology in Modern Sports Training}

\subsection{Advantages}

(1) It can ensure thateach index in different periods of time nodes do not repeat, which can realize the diversification of time;

(2) Based on the collected data, different types of results can be achieved through different ways.

\subsection{Disadvantages}

(1) Computer virtual technology in modern sports system is not well developed which posed a serious impact on its application;

(2) The application and research demand for a large number of data, in this sense, the data collection is far from satisfying people's need.

\subsubsection{Opportunities}

The application of computer virtual technology in modern sports, in fact, is an effective way to solve the problem of high load construction costs and realize the goal that other systems cannot reach.

(1) The computer virtual technology has the characteristics of data collection, data

Processing and convenience, which makes it more convenient for the application of modern sports;

(2) The high priorities towards sports career throughout the country as well as the high degree of concern for the physical education have laid the solid foundation for the development of virtual technology in sports 


\section{Research on Modern Sports Training Model Based on Computer Virtual Technology}

\subsection{Simulation Assumption}

(1) When arms are stretched, it is the largest movement of the human body space and after the rotation of the upper body, the space is formed.

(2) When the normal activity space is for the arm straight and the upper body does not move, the space is formed.

(3) When the best activity space is for bend and stretch of arms, it is the most comfortable activity space.

(4) The angle range involved in the model is specified in this paper.

\subsection{Model Preparation}

(1) Motion data information collection

Before build up the model, the information acquisition on motion data is needed. At present, the main methods are as follows:

Table 1. The Mode of Gaining the Body's Information

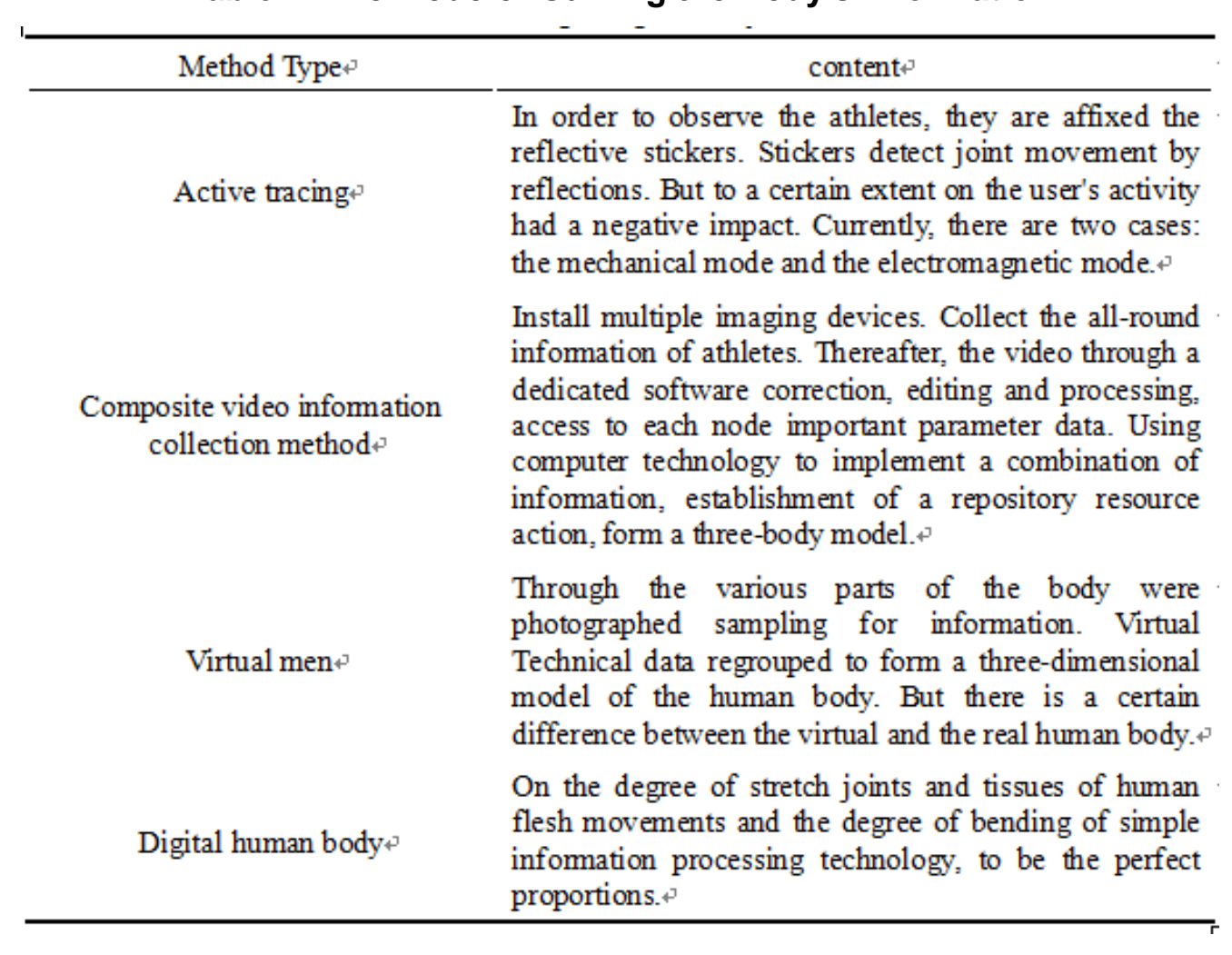

Taking into account the characteristics of the above methods, and combining with the existing technology, a multi position camera information collection method have been used. Based on mechanical principle, electromagnetic principles, acoustical principle as well as optical principle, kinematic method in physics and forward kinematics and inverse kinematics in dynamics are used in the model. The model sets joint animation and the joint rotation angle in order to complete the collection of relevant information.

(1) The main joint rotation angle of the human body 
Human activities mainly consider two aspects: the distance of physical activities and the activity angle of the body. Based on a large number of data, the results can be obtained as follows:

Table 2. Human Motion Joint Angle Reference Data

\begin{tabular}{|c|c|c|c|c|c|c|}
\hline \multirow[b]{2}{*}{ joint } & \multicolumn{2}{|c|}{ X } & \multicolumn{2}{|c|}{$\mathrm{Y}$} & \multicolumn{2}{|c|}{ Z } \\
\hline & bend & stretch & $\begin{array}{c}\text { Outer } \\
\text { surround }\end{array}$ & $\begin{array}{c}\text { Within } \\
\text { surround }\end{array}$ & $\begin{array}{l}\text { Outer } \\
\text { stretch }\end{array}$ & $\begin{array}{l}\text { Within } \\
\text { Collapse }\end{array}$ \\
\hline spine & 95 & 55 & 45 & 45 & 45 & 45 \\
\hline arm & 135 & 35 & 90 & 90 & 35 & 180 \\
\hline stride & 140 & 0 & - & - & - & - \\
\hline
\end{tabular}

\subsection{Model Building}

(1) Spinal motion model

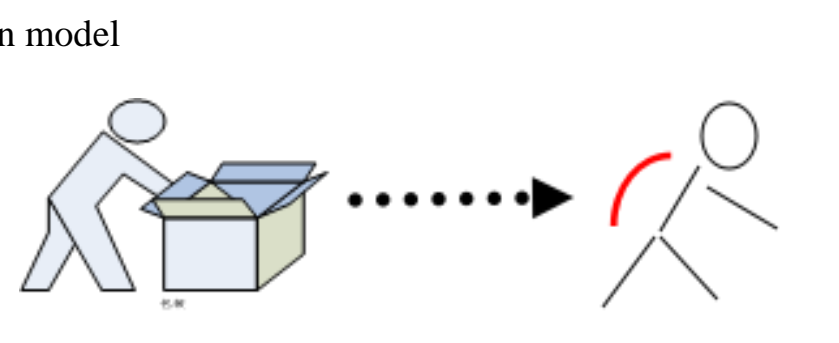

Figure 3. Spinal Motion Diagram

As an important key to the human body, the spine plays a very important role in the movement and the change of direction of the body. According to the swinging angle and distance theory in physics, the human spine model can be obtained as follows:

$$
Q=-\omega(\xi) Q_{L}=-p \xi \frac{\left[Q_{X} 0, Q_{Z}\right]^{T}}{\sqrt{Q_{X}^{2}+Q_{y}^{2}}}
$$

$\omega(\xi)$ is the planar projection of $Q$ on $\mathrm{x}-\mathrm{y}, p$ is the specified value

$0.1 \mathrm{~m} / 120 \mathrm{deg} \cdot \xi$ stands for the rotation angle of the mode. $Q$ stands for the planar projection of $Q$ on $X-Z$, namely $Q=D-D^{\prime}$.

(2) Arm motion model
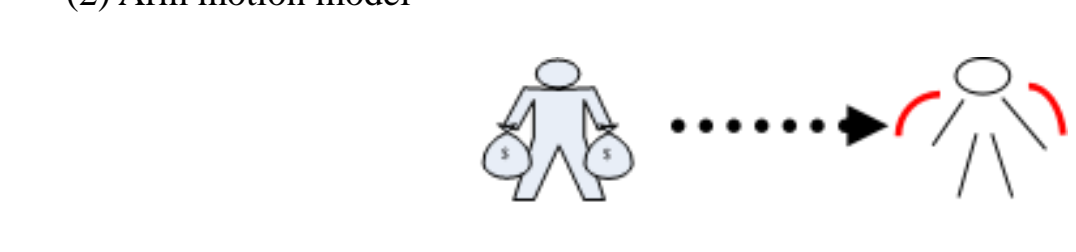

Figure 4. Arm Motion Diagram

The arm motion model is iterated through the dynamical square module, and taking into account of the condition of the arm under different conditions, such relation formula can be obtained:

$$
J=-V_{t}\left(L-L_{h}\right)-V_{c} L
$$


In the formula, $L$ stands for the initial position of the end of the arm. $L_{h}$ stands for the ending position of the end of the arm and $V$ stands for the gain error.

(3) Stepping motion model

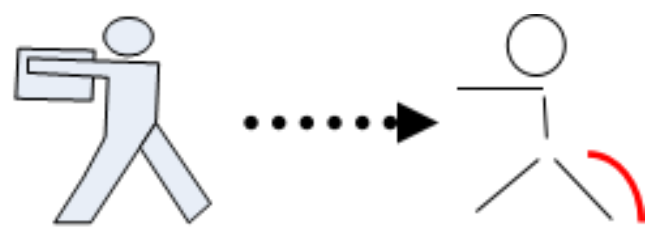

Figure 5. Stepping Motion Diagram

Based on the constraint formula of closed chain structure, and by using the forward kinematics theory in physics and feedback control mechanism, arm movement model has been obtained:

$$
U=\left(E_{A}-E_{B}\right)\left(\begin{array}{l}
\xi_{N} \\
\xi_{M}
\end{array}\right)=\left(\frac{\partial A}{\partial M}-\frac{\partial B}{\partial N}\right)\left(\begin{array}{l}
\xi_{M} \\
\xi_{B}
\end{array}\right)
$$

In the formula, $E$ stands for Jacobian matrix of arm movement model. $\xi$ stands for rotation angle. Jacobian matrix is a kind of matrix produced by the ratio of deviation.

(4) Human motion model

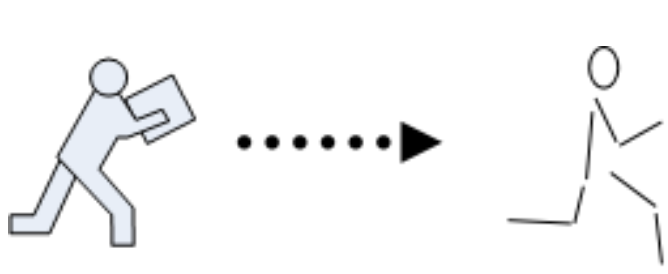

\section{Figure 6. Hưman Motion Diagram}

Three-dimensional model of numan body which mainly includes human body joints, human bones and gravity center of human body is constructed, which divided human body into three levels of muscle, skeleton and skin. Set the position of $I$ in a node in rectangular coordinate system $R . x, y, z$ stands for three parameters, $I^{\boldsymbol{R}}$ is the reference coordinate of the rectangular coordinate system $R$. It can be shown as follows:

$$
I^{R}=\left[\begin{array}{l}
x \\
y \\
z
\end{array}\right]
$$

\footnotetext{
V is based on $I^{R}$ and taking into account of human body movement direction, namely the rotation angle of $x, y, z$, by using the relations between the variations of angle in physics, The rotation matrix is obtained by the solution of the mathematical method and the characteristic of the direction cosine of the coordinate system. It can satisfy the need of changing the angle of motion in the human body movement model and improving the original model, thus Matrix-Vector can be expressed by $\boldsymbol{E}$
} 


$$
I^{\varepsilon}=[X, Y, Z]=\left[\begin{array}{lll}
\varepsilon_{11} & \varepsilon_{12} & \varepsilon_{13} \\
\varepsilon_{21} & \varepsilon_{22} & \varepsilon_{23} \\
\varepsilon_{31} & \varepsilon_{32} & \varepsilon_{33}
\end{array}\right]
$$

$I^{T}$ is based on $I^{R}$. Take the change of different movement in the process of the translation and rotation into consideration, spatial switching can be realized. In four-dimensional space, $I_{1}=(0,0,0,1)^{T}$ is the origin of coordinates. $I_{2}=(0,0,0,1)^{T}$ stands for the four-dimensional space in $O X$ axis. $I_{3}=(0,0,0,1)^{T}$ stands for the four-dimensional space in $O r$ axis. $I_{4}=(0,0,0,1)^{T}$ stands for the four-dimensional space in $O Z$ axis. Transfer matrix $H$ can be shown as follows:

$$
H=\left[\begin{array}{llll}
1 & 0 & 0 & \alpha \\
0 & 1 & 0 & \beta \\
0 & 0 & 1 & \lambda \\
0 & 0 & 0 & 1
\end{array}\right]
$$

There is more than one form of human movement. Considering the variation of movement generated by the translation and rotation of human yariation matrix $\Gamma^{\boldsymbol{T}}$ can be obtained as follows: punctual

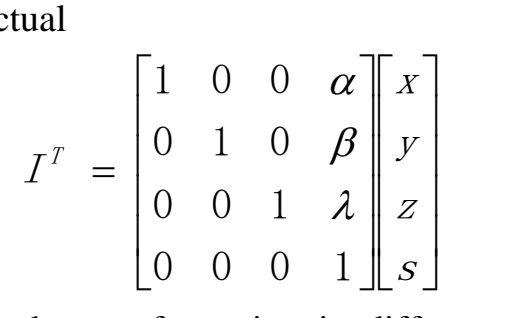

After analyzing the body transformation in different coordinate system, namely the rotation angle $\mu$ rotate around $\mathrm{X}$ axis, and then rotate $\nu$ degree around $Y$ axis and then rotate $O$ degree around $Z$ axis thus the model matrix $I^{*}$ of modern physical training in virtual technology is obtained.

$$
I^{\prime}=\left[\begin{array}{cccc}
\cos \mu & \sin \mu & 0 & 0 \\
0 & 0 & 1 & 0 \\
0 & 0 & 0 & 1
\end{array}\right]\left[\begin{array}{cccc}
\cos v & 0 & \sin v & 0 \\
0 & 1 & 0 & 0 \\
-\sin v & 0 & \cos v & 0 \\
0 & 0 & 0 & 1
\end{array}\right]\left[\begin{array}{cccc}
\cos o & -\sin o & 0 & 0 \\
\sin o & \cos o & 0 & 0 \\
0 & 0 & 1 & 0 \\
0 & 0 & 0 & 1
\end{array}\right]
$$

\section{Simulation Experiment on Human Body}

Human simulation experiment in virtual technology is an important demand of reform in modern sports career, which has the core characteristics in modern education. The relevant objectives and targets raised in this experiment have realized the goal of the construction of campus, laboratory and teaching resource library. By focusing on autonomous learning and thinking in information-based society, it meets the application and optimization of teaching resources as well as guarantee the pushing role in high quality resources. With the characteristics of integration and combination, the reform of experimental teaching has been promoted, which has brought new impetus to the cause of modern sports. 


\subsection{Platform Construction}

Before realizing the human body simulation experiment, the construction of the experiment platform is the first step. First of all, the virtual software and application system need to be prepared before the experiment and the computer source code should be written before and a reasonable agreement also needs to be constructed before. Secondly, complete the parameter configuration of the software system, on the one hand, complete the internal parameters, on the other hand, and complete the peripheral parameters. Finally, the trial operation in virtual environment should be conducted. By using the well-established code and parameters, system operation can be operated, and the results of the experiment to can be tested so as to complete the test of the data package.

\subsection{Simulation Experiment}

The realization of human body simulation experiment, which takes the human lumbar joint as the center is conducted on the basis of the coordinate system. First of all, get the sub node list of the current joint point and do a series of matrix transformation and then rotation transformation of the joint point shall be made. On the basis of the world coordinate system, the model can be drawn. Observe that whether there exists other child node. If there do exist, this child node should be transformed, otherwise, continue to draw a picture of human motion, and then set up the target experiment database. After the simulation experiment, the loopholes in physical exercise can be corrected and by comparing with the previous exercise, the results can be obtaned. Human Simulation work flow chart is as follows:

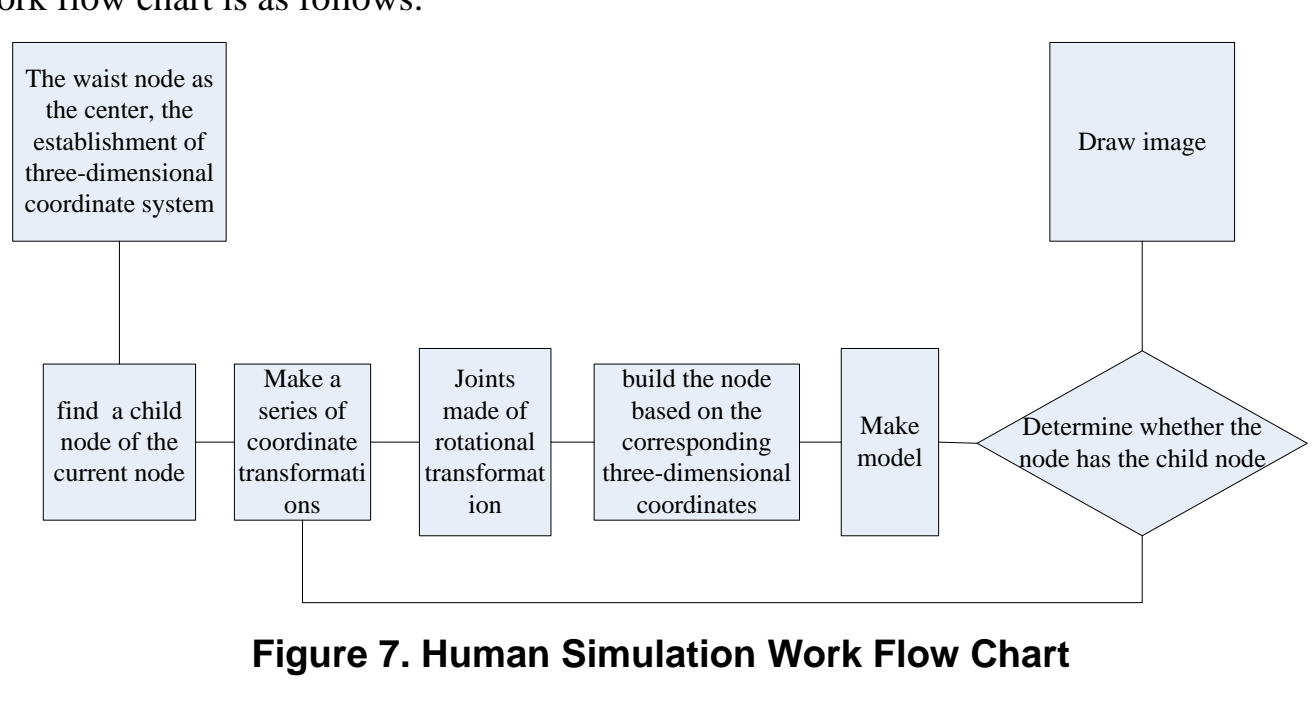

The notmalization of human spine, arms, step and the human body movement through the simuration of the model have been improved and it has made positive effect on the normalization of the movement and positive effect all reached more than $50 \%$. The results are detalled in table:

Table 3. Simulation Results Table

\begin{tabular}{cccccc}
\hline mode & before & after & result $(\%)$ & effect \\
\hline Spinal motion model & 0.2541 & 0.1021 & & $+59.82 \%$ & positive \\
Arm motion model & 0.2517 & 0.0568 & $+77.43 \%$ & positive \\
Stepping motion model & 0.3297 & 0.0157 & $+95.23 \%$ & positive \\
Overall body motion model & 0.5124 & 0.1245 & $+75.70 \%$ & positive \\
\hline
\end{tabular}


In order to clearly show the difference of the model on human motion, the above situation can be generalized as follow. As seen from the chart, the comparison chart of the correction before and after human motion is as follows, among which the correction on stepping motion is most effective which reaching more than $95 \%$, and then followed by the arm movement model and the human body model, all reached more than $70 \%$ and the lowest level of correction is the spinal motion model, the effect of more than $50 \%$. The ranking of the positive effect on human movement from high to low is respectively the stepping motion model, arm movement model, human body model and spinal motion model. but

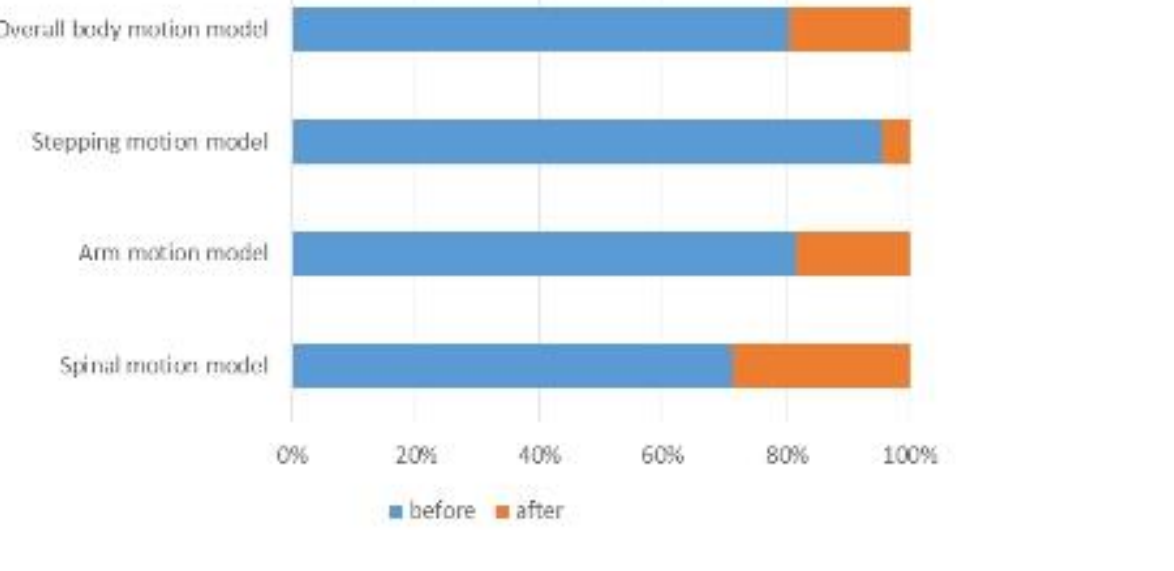

Figure 8. The Human Motion Model Before and After Comparison Chart

\section{Conclusion}

The development of science and technology is leading the trend of the times and the application of information pechnology has complied with the development of history. In response to the policy of national seience and technology research and development and meet the requirement of people a healthier body condition, by collecting human motion information throagh the relevant principles of physical, and taking into account the horizontal hisplacement and rotation angle, the model of the spine arms, step and the human body is establishedis this paper. Through the analysis of the technology of computer and information, angle and way of movement have been corrected. By comparing the reference model of human motion before and after, the conclusion that modern sports training can be improved by using the virtual computer technology. The construction of the model has largest impact on stepping, and followed arms human body and spine. In general, the application of computer virtual technology in the modern sports career has made a contribution to the construction of the sports industry in our country, and if also has an important influence to the improvement of the physical quality of the whole society.

\section{Development Trend}

Computer virtual technology is a comprehensive application technology of digital processing based on reality. It is a combination of various disciplines which has not only applied in practical use but a combination of various technologies. At present, only taking universities and colleges into consideration, until now, it has more than 200 schools established the system of experiment center. A more interesting interactive and effective way of virtual teaching system has been designed. Especially after the "Internet plus" theory proposed and in response to the strategy of making China powerful through 
science and technology and the strategy of develop the country through physical education, especially for the application of computer virtual technology in modern sports, a broad platform which can provide a more scientific method and reduce more work has been built. The main developing points and main developing fields of computer virtual technology are as follows:

\subsection{The Main Developing Points}

Computer virtual technology in modern sports training methods possesses great prospects. With the extensive application of the technology, adhering to the virtual manufacturing system theory, virtual environment theory, practical manufacturing and virtual manufacturing theory of binding theory and virtual technology related equipment reengineering theory, a qualitative leap has been achieved and the main trend is: computer virtual technology, engineering application and rapid prototyping mechanism of computer virtual technology. The more prominent potential is the combination of human and machine intelligence, that is, by experiencing and analyzing the human emotions and feelings, the computer can understand the intention of human activities and thus a virtual human body can be created. Such synthetic body has the ability to sense sound and understand human's intent.

\subsection{The Main Development Field}

As the key scientific research project, the computer virtual technology is not only widely used in modern sports, but also plays a key role to the future development. Computer virtual technology can not only) be used in military affairs, education, architecture and medicine, virtual enyironment and real-time monitoring, but also be helpful in high quality simulation experiment. It can solve the problems of computer at the same time, it also become the best assistant of the human life. At present, the computer remote simulation technology is alsoin study.

\subsection{Main Challenge in Development}

Due to the diversity and generalization of the knowledge form, people have deepened the cognition of virtual technology; meanwhile, people also have faced new opportunities and challenges. The first challenge comes from technology and media, which mainly manifested in the development of technology and the times, the training of talents, a higher requirement towards the method and mode of virtual technology and virtual technology. Secondly, the cognition of modern sports and the idea of computer virtual technology are facing the challenge. The depth integration in computer virtual technology and modern sport teaching, to a certain extent, creates a new sense of education and teaching. For example, transform knowledge teaching into the ability training.

\subsection{The Construction and Practice of Virtual Technology in the Future Teaching of Physica) Education}

Computer virtual technology has very important guiding significance for the future teaching of physical education. First of all, users and students can achieve their own needs according to their personal preferences and through independent experiments, autonomous learning, a way of combining of theory and practice can be achieved. Secondly, due to project professionalism, dangerousness and destructiveness as well as the security risks and legal risks in the process of practice, the original plan is restricted, however, virtual technology has the strength to make up for the disadvantages. Third, by using computer virtual technology in the process of virtual simulation, the cost of investment and construction projects can be reduced. Fourth, virtual technology can achieve the goal of sharing resources, breaking the limitations of the study, which is 
conducive to the comprehensive study and application of users thus can greatly improving the efficiency of energy utilization and benefits.

\section{References}

[1] D. E. Orin and W. W. Schrader, "Efficient computation of the jacobian for robot manipulators", The International Journal of Robotics Research, vol. 3, no. 4, (1984), pp. 66-75.

[2] J. K. Hodgins, P. K. Sweeney and D. G. LawTence, "Generating natural looking motion for computer animation", Graphics Interface, (1992), pp. 265-272

[3] J. S Miller, "Digital humans in the simulated Product life cycle", IIE Solutions. Noerorss, vol. 30, no. 155.3, (1998), pp. 6-24.

[4] N. Bdaler, C. B. PhilliPs and B. L. Webber, "Simulating humans: Computer graphics animation and control", Oxford University Press, New York, Oxford, (1993).

[5] S. J. Berhrens. "A conceptual analysis and historical over-view of information literacy", College and research libraries, no. 4, (1994), pp. 309-322.

[6] M. D. Roblyer, "Integrating Educational Technology into Teaching", Xian: Pearson Education Asia Limited and Shanxi Normal University Press, vol. 4, (2005).

[7] K. Suncheol and K. Jung, "Real-time upper limb motion prediction from noninvasive biosignals for physical human-machine interactions", 2009 IEEE International Conference on Systems, Man and Cybernetics, (2009), pp. 847-852.

[8] M. Pidd, "Introduction to computer simulation Proceeding of WSC94" vol. 94, pp. 7-14.

[9] A. M. Law and W. D. Kelton, "Simulation M odeling and Analysis", McGraw-Hill, inc (1991).

[10] M. D. Roblyer, "Integrating Educational Technology into Teaching", Xian Pearson Education Asia Limited and Shanxi Normal University, Press, vol. 4, (2005).

[11] L. Youquan, "Study on Acceleration Techniques of Physically Based Computer Animation", Beijing: ISCAS, (2005).

[12] J. Wiley, "Taking the next step. Assembly simulation", Modern Manufacturing, vol. 4, (1997), pp. 17-34.

[13] Z. Junhong, S. Shaojun, Z. Yong, G. Hongge and C. Xiaoming, "Torsion Oscillation Characteristics of Rotary Shafts Based on Torsion and Bending Coupled Vibration".

[14] T. Kozik and M. Simon, "Preparing and managing the remote experiment in education", 15th International Conference on Interactive Collaborative Learning. Villach: IEEE, (2012), pp. 26-28.

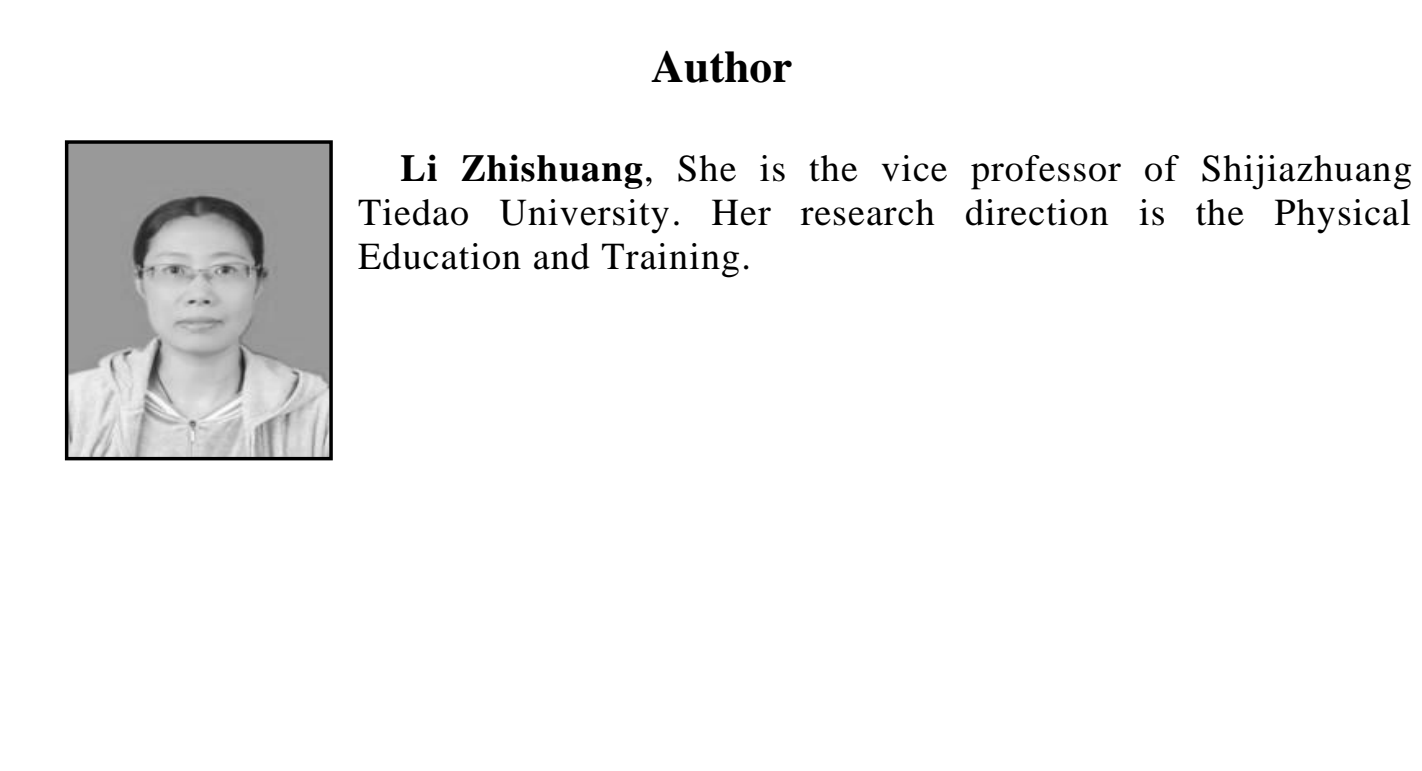

